\title{
Maps into the torus and minimal coincidence sets for homotopies
}

by

\author{
D. L. Gonçalves (São Paulo) and M. R. Kelly (New Orleans, LA)
}

\begin{abstract}
Let $X, Y$ be manifolds of the same dimension. Given continuous mappings $f_{i}, g_{i}: X \rightarrow Y, i=0,1$, we consider the 1-parameter coincidence problem of finding homotopies $f_{t}, g_{t}, 0 \leq t \leq 1$, such that the number of coincidence points for the pair $f_{t}, g_{t}$ is independent of $t$. When $Y$ is the torus and $f_{0}, g_{0}$ are coincidence free we produce coincidence free pairs $f_{1}, g_{1}$ such that no homotopy joining them is coincidence free at each level. When $X$ is also the torus we characterize the solution of the problem in terms of the Lefschetz coincidence number.
\end{abstract}

0. Introduction. For a pair of maps $(f, g): X \rightarrow Y$ denote by Coin $(f, g)$ the set $\{x \in X \mid f(x)=g(x)\}$. Assume $X$ and $Y$ to be compact manifolds of the same dimension, in which case this set is generically a finite set of points. Now suppose that $\left(f_{1}, g_{1}\right),\left(f_{2}, g_{2}\right)$ are homotopic as a pair of maps, and that $\# \operatorname{Coin}\left(f_{1}, g_{1}\right)=\# \operatorname{Coin}\left(f_{2}, g_{2}\right)=\operatorname{MC}\left[f_{1}, g_{1}\right]$, where $\operatorname{MC}[f, g]$ denotes the mininal number of coincidence points occurring among all pairs $\left(f^{\prime}, g^{\prime}\right)$ homotopic to $(f, g)$. A natural question is the following: Can one find a pair $H, G$ of homotopies from $f_{1}$ to $f_{2}$ and $g_{1}$ to $g_{2}$, respectively, such that \#Coin $(H(\cdot, t), G(\cdot, t))=\mathrm{MC}\left[f_{1}, g_{1}\right]$ for all $t \in[0,1]$ ? We refer to this as the minimal coincidence problem.

A variation of a special case of this question was raised by H. Schirmer in [8], in particular, the fixed point problem, where we take $X=Y$, both $g_{1}$ and $g_{2}$ are the identity and the homotopy $G$ remains constant at the identity. For self-maps of $S^{1}$ it can be shown that the fixed point case has an affirmative answer. On the other hand, M. Kelly [6] has provided examples of manifolds of all dimensions $\geq 2$ and self-maps $f_{1}, f_{2}$ which are homotopic, fixed point free but not freely homotopic, i.e. they cannot be connected by a homotopy $H$ which is fixed point free. It is not known if these provide counterexamples to the minimal coincidence problem be-

2000 Mathematics Subject Classification: 55M20, 57M05. 
cause of the assumption that $G$ is the identity in the fixed point problem.

In the paper [2] Dimovski and Geoghegan develop primary and secondary geometric obstructions to removing circles of fixed points occurring in homotopies when $X$ is a PL manifold of dimension at least 4 . They show that the vanishing of the obstructions implies that fixed points can be removed. For the examples in [6] the primary obstruction is nonzero. Results in [2] were generalized by Jezierski in [5]. Later, Geoghegan and Nicas [3] using Hochschild Homology gave a 1-parameter fixed point theory, analogous to classical Nielsen theory, defined for finite CW complexes. For $X$ a PL manifold of dimension at least four this theory gives exactly the primary obstruction of [2].

The purpose of this work is to study the minimal coincidence problem in low dimensions. More specifically, we consider the setting where the target $Y$ is a torus of dimension either 1 or 2 . We present several results. In particular, when the domain and target are both the 2-torus, we see in Theorems 1.4 and 3.4 that the solution depends on the value of the Lefschetz coincidence number $\Lambda(f, g)$ of a given pair $(f, g)$ of maps. It is shown in [1, Section 6] that for the torus $|\Lambda(f, g)|=\mathrm{MC}[f, g]$. Throughout this paper the letter $T$ will be used to denote the 2-dimensional torus.

TheOREM 1.4. Let $\left(f_{0}, g_{0}\right): T \rightarrow T$ be a pair of maps which is coincidence free. Then there is a countable family of maps $\left(f_{n}, g_{n}\right)$, each coincidence free and homotopic to $\left(f_{0}, g_{0}\right)$, so that for any two pairs of maps $\left(f_{m}, g_{m}\right),\left(f_{n}, g_{n}\right)$ in the family with $m \neq n$ each homotopy between them has coincidence points.

TheOREM 3.4. Let $\left(f_{1}, g_{1}\right),\left(f_{2}, g_{2}\right): T \rightarrow T$ be homotopic pairs of maps, with $\left|\Lambda\left(f_{i}, g_{i}\right)\right|=n$ for $i=1,2$ and $n \neq 0$. If $\# \operatorname{Coin}\left(f_{i}, g_{i}\right)=n$ for $i=1,2$ then there is a pair of homotopies $H, G$ between $\left(f_{1}, g_{1}\right)$ and $\left(f_{2}, g_{2}\right)$ such that $\# \operatorname{Coin}(H(\cdot, t), G(\cdot, t))=n$ for all $t \in[0,1]$.

As a comparison we also consider the 1-dimensional situation. Here the result is slightly different.

THEOREM 4.2. Let $\left(f_{1}, g_{1}\right),\left(f_{2}, g_{2}\right): S^{1} \rightarrow S^{1}$ be homotopic pairs of maps, each pair having $\left|\Lambda\left(f_{i}, g_{i}\right)\right|$ coincidence points. Then there is a pair of homotopies $H, G$ between $\left(f_{1}, g_{1}\right)$ and $\left(f_{2}, g_{2}\right)$ such that $\# \operatorname{Coin}(H(\cdot, t), G(\cdot, t))=$ $\left|\Lambda\left(f_{i}, g_{i}\right)\right|$ for all $t \in[0,1]$.

REMARK. Because the torus admits a group structure, slightly stronger versions of Theorems 3.4 and 4.2 can be proved. Namely, given an arbitrary homotopy $G$ one can always find a homotopy $H$ such that the pair $(H, G)$ satisfies the conclusion of the theorems. 
The remainder of this paper is organized as follows. In Section 1 we consider coincidence point free pairs of maps $(f, g): T \rightarrow T$. We first obtain the result for the corresponding root problem for maps of degree zero. As a consequence we prove the main result of the section, Theorem 1.4. In Section 2 we consider coincidence point free pair of maps $(f, g): S_{h} \rightarrow T$ for maps from a closed surface of genus $h>1$ to the torus. The main result is Theorem 2.2. In Section 3 we consider the case of non-coincidence free selfmaps of the torus. The main result for coincidence is given in Theorem 3.4. Finally, the parallel results in dimension one are proved in Section 4.

We conclude the introduction with the comment that an analog of these results for higher-dimensional tori is unknown. Both, the algebra needed for an analog of Theorem 1.4 seems more difficult, and the methods used to prove 3.4 are restricted to dimension two.

1. Coincidence free self-maps on the torus. We begin by considering the particular case of roots, where coincidence free translates into maps having degree equal to zero. Then, using the multplication on the torus, we obtain the corresponding result for the general case of coincidence, and also as a particular case, the fixed point case. Let $1 \in T$ denote the multiplicative identity.

We start with one algebraic lemma regarding conjugate elements in a free group. Let $F(a, b)$ be the free group on two generators $a, b$, and $B=[a, b]$, the commutator of $a$ and $b$.

Lemma 1.1. Let $w \in F$. Then $w B^{2 n}$ and $w B^{2 m}$ are not conjugate for $m, n$ being different integers.

Proof. Suppose that there exists $\xi \in F$ such that $w B^{2 n}=\xi w B^{2 m} \xi^{-1}$. This implies that $B^{2 n-2 m}=B^{-2 m} w^{-1} \xi w B^{2 m} \xi^{-1}=\left[B^{-2 m} w^{-1}, \xi\right]$. By [4] we know that an even power of $B$ cannot be written as a single commutator.

Proposition 1.2. Suppose $f_{0}: T \rightarrow T$ is such that $1 \notin f_{0}(T)$. Then there exist a countable family of maps $f_{n}$, each homotopic to $f_{0}$, such that $1 \notin f_{n}(T)$ and for any two maps $f_{m}, f_{n}$ in the family with $m \neq n$ each homotopy between them has a root at 1 .

Proof. By hypothesis we have $f_{0}: T \rightarrow T$ with image missing 1 . Let $1 \in T$ be the base point of $T$ and let $f_{0}(1)=y_{0}$ be the base point of $T \backslash\{1\}$. Let $e_{1}, e_{2} \in \pi_{1}(T,\{1\})$ be the canonical generators and $a, b \in \pi_{1}\left(T \backslash\{1\}, y_{0}\right)$ two generators of this free group. Set $f_{0 \#}\left(e_{1}\right)=w_{1}, f_{0 \#}\left(e_{2}\right)=w_{2}$. Since $\left[e_{1}, e_{2}\right]=0$, it follows that the commutator $\left[w_{1}, w_{2}\right]$ is trivial in $T \backslash\{1\}$. This implies that there is a word $w$ such that $w_{1}=w^{r}, w_{2}=w^{s}$. Now define $f_{n}^{0}: S^{1} \vee S^{1} \rightarrow T \backslash\{1\}$ as follows: $f_{n}^{0}\left(e_{1}\right)=\left(w B^{2 n}\right)^{r}$ and $f_{n}^{0}\left(e_{2}\right)=\left(w B^{2 n}\right)^{s}$. 
The elements defined by the homotopy classes of the loops $\left(w B^{2 n}\right)^{r}$ and $\left(w B^{2 n}\right)^{s}$ commute because these words are powers of the same word. So $f_{n}^{0}$ extends to a map $f_{n}^{\prime}: T \rightarrow T \backslash\{1\}$. Let $f_{n}$ denote the composition of $f_{n}^{\prime}$ with the inclusion $T \backslash\{1\} \subset T$.

All of the maps $f_{n}$ are homotopic because in $\pi_{1}(T)$ we have $w^{r}=$ $\left(w B^{2 n}\right)^{r}$ and $w^{s}=\left(w B^{2 n}\right)^{s}$. We claim that $f_{m}^{\prime}, f_{n}^{\prime}$ are not homotopic when $m \neq n$. Otherwise, the words $\left(w B^{2 m}\right)^{r},\left(w B^{2 n}\right)^{r}$ and $\left(w B^{2 m}\right)^{s},\left(w B^{2 n}\right)^{s}$ are in the same conjugacy class. More precisely, there exists $\xi$ such that $\left(w B^{2 n}\right)^{r}=\left(\xi w B^{2 m} \xi^{-1}\right)^{r}$ and $\left(w B^{2 n}\right)^{s}=\left(\xi w B^{2 m} \xi^{-1}\right)^{s}$. By [7], Ex. 3, page 41 , we must have $\left(w B^{2 n}\right)=\left(\xi w B^{2 m} \xi^{-1}\right)$. By Lemma 1.1, this is not possible and the result follows.

The above result will be used to prove the analogous results in the fixed point and coincidence cases.

THEOREM 1.3. Let $g_{0}: T \rightarrow T$ be fixed point free. Then there is a countable family of maps $g_{n}$, each fixed point free and homotopic to $g_{0}$, so that for any two maps $g_{m}, g_{n}$ in the family with $m \neq n$ each homotopy between them has fixed points.

Proof. Let $f_{0}(x)=g_{0}(x) \cdot x^{-1}$. This is a map $f_{0}: T \rightarrow T$ with $1 \notin f_{0}(T)$. Consider the family $f_{n}$ defined in Proposition 1.2. Let $g_{n}(x)=f_{n}(x) \cdot x$. Then certainly $g_{n}$ is fixed point free. Finally, $g_{m}$ and $g_{n}$ cannot be joined by a fixed point free homotopy, otherwise $f_{m}$ and $f_{n}$ could be joined by a root free homotopy.

TheOREM 1.4. Let $\left(f_{0}, g_{0}\right): T \rightarrow T$ be a pair of maps which is coincidence free. Then there is a countable family of maps $\left(f_{n}, g_{n}\right)$, each coincidence free and homotopic to $\left(f_{0}, g_{0}\right)$, so that for any two pairs of maps $\left(f_{m}, g_{m}\right),\left(f_{n}, g_{n}\right)$ in the family with $m \neq n$ each homotopy between them has coincidence points.

Proof. Let $h_{0}(x)=g_{0}(x) \cdot f_{0}(x)^{-1}$. This is a map $h_{0}: T \rightarrow T$ with $1 \notin h_{0}(T)$. Consider the family $h_{n}$ defined in Proposition 1.2. Let $g_{n}(x)=$ $h_{n}(x) \cdot f_{0}(x)$ and $f_{n}=f_{0}$. Since $h_{n}(x) \neq 1$ for all $x$ we see that $\left(f_{n}, g_{n}\right)$ is coincidence free. Also, the pairs $\left(f_{n}, g_{n}\right)$ and $\left(f_{m}, g_{m}\right)$ cannot be joined by a coincidence point free homotopy, otherwise $h_{n}$ and $h_{m}$ could be joined by a root free homotopy.

\section{Coincidence free maps from an orientable surface to the} torus. We now look at the situation when the domain for our maps is the closed orientable surface of genus $h \geq 2$, which we denote by $S_{h}$. As in Section 1, we first consider the particular case of roots.

Proposition 2.1. Suppose $f_{0}: S_{h} \rightarrow T$ is such that $1 \notin f_{0}\left(S_{h}\right)$. Then there exists a countable family of maps $f_{n}$, each homotopic to $f_{0}$, so that 
$1 \notin f_{n}\left(S_{h}\right)$ and for any two maps $f_{m}, f_{n}$ in the family with $m \neq n$ each homotopy between them has a root at 1 .

Proof. Let $e_{1}, \ldots, e_{2 h}$ be generators for $\pi_{1}\left(S_{h}, x_{0}\right)$. Let $w_{i}=f_{0 \#}\left(e_{i}\right)$, where each $w_{i}$ is a word in the free group $\pi_{1}\left(T \backslash\{1\}, f\left(x_{0}\right)\right)$ on 2 generators. Since $f_{0}$ is root free, the $w_{i}$ 's satisfy the equation $\left[w_{1}, w_{2}\right] \ldots\left[w_{2 h-1}, w_{2 h}\right]=1$. Let us assume that $\left[w_{1}, w_{2}\right] \neq 1$. If not, an argument similar to Proposition 1.2 can be used to construct the required maps. Let $\left[w_{1}, w_{2}\right]$ be denoted by $K$. For each integer $n$ define a map $f_{n}$ from $S_{h}$ to $T \backslash\{1\}$ such that $f_{n \#}\left(e_{i}\right)=w_{i}, i>2$, and $f_{n \#}\left(e_{i}\right)=K^{n} w_{i} K^{-n}, i=1,2$. As maps into $T$ they are all homotopic for either of two reasons: because $K$ belongs to the kernel of $\pi_{1}(T \backslash\{1\}) \rightarrow \pi_{1}(T)$ or because $\pi_{1}(T)$ is abelian. On the other hand, we claim that any two such maps, as maps from $S_{h}$ into $T \backslash\{1\}$, cannot be homotopic. The proof is completed by assuming $f_{n}$ and $f_{m}$ are homotopic and deriving a contradiction.

Assume $f_{n}$ and $f_{m}$ are homotopic. Then the induced homomorphisms on the fundamental groups are conjugate. Thus, there exists $\theta$ such that $f_{n \#}\left(e_{i}\right)=\theta f_{m \#}\left(e_{i}\right) \theta^{-1}$ for all $i$. Now, $\theta=1$ is not possible because this would imply $K^{n} w_{i} K^{-n}=K^{m} w_{i} K^{-m}$ for $i=1,2$. So $K^{m-n}$ commutes with $w_{i}$ for $i=1,2$. Hence there are subgroups $H_{1}$ and $H_{2}$ of rank one which contain $K^{m-n}, w_{1}$ and $K^{m-n}, w_{2}$ respectively. But in a free group the subgroup generated by $H_{1}$ and $H_{2}$ is also free and has rank at most two. Because both contain the common nontrivial element $K^{m-n}$, its rank must be one. This implies that $\left[w_{1}, w_{2}\right]=1$. So we must have $\theta \neq 1$ and $\theta$ commutes with each $w_{i}$ for $i>2$. Therefore all the $w_{i}$ 's for $i>2$ belong to a single subgroup of rank one and it follows that $\left[w_{2 i-1}, w_{2 i}\right]=1$ for $i=2, \ldots, h$. But this implies that $\left[w_{1}, w_{2}\right]=1$, which is a contradiction.

As a consequence of Proposition 2.1 we obtain the following theorem. Its proof is the same as that of Theorem 1.4.

TheOREM 2.2. Let $\left(f_{0}, g_{0}\right): S_{h} \rightarrow T$ be a pair of maps which is coincidence free. Then there is a countable family of maps $\left(f_{n}, g_{n}\right)$, each coincidence free and homotopic to $\left(f_{0}, g_{0}\right)$, so that for any two pairs of maps $\left(f_{m}, g_{m}\right),\left(f_{n}, g_{n}\right)$ in the family with $m \neq n$ each homotopy between them has coincidence points.

We conclude this section with the comment that the extension of Proposition 2.1 to the nonzero degree case, and hence Theorem 2.2 to the noncoincidence free case, is apparently more subtle. A difficulty arises in the method of proof given in Proposition 2.1 as soon as $f_{0}^{-1}(1) \neq \emptyset$, and also the methods used in this section do not generally apply. This case provides an interesting problem for further investigation into the study of the existence of minimal homotopies for coincidences in dimension two. 
3. Maps which are not coincidence free. In this section we consider a pair of maps $(f, g): T \rightarrow T$ where the pair cannot be made coincidence free. As in Section 1, we recall that when the target is the torus, once we obtain the results for the root case, the results for the coincidence problem and for the fixed point problem follow. For the root problem we are now concerned with the case when the degree is nonzero, and so our maps are no longer root free. We first consider the case when the degree is \pm 1 .

Lemma 3.1. Let $f: T \rightarrow T$ be a map of degree $d$, where $d= \pm 1$. Suppose that $y_{1}, \ldots, y_{l}$ is a finite set of points in $T$ such that $f^{-1}\left(y_{i}\right)$ is a single point for each $i$, and further, the local degree at each $f^{-1}\left(y_{i}\right)$ is $d$. Then we can deform $f$ relative to $f^{-1}\left(y_{1}\right), \ldots, f^{-1}\left(y_{l}\right)$ to a homeomorphism $f^{\prime}$ such that the homotopy $H$ between $f$ and $f^{\prime}$ satisfies $\# H(\cdot, t)^{-1}\left(y_{i}\right)=1$ for all $t \in[0,1]$ and $i=1, \ldots, l$.

Proof. For simplicity of notation we assume $i=1$ and set $y=y_{1}$. The proof for $i>1$ is identical. Let $N$ be a small neighborhood of $y$ with $N \backslash y$ foliated by circles $\gamma_{t}, 0<t \leq 1$. Let $M$ be a small neighborhood of $f^{-1}(y)$ with $M \backslash f^{-1}(y)$ foliated by $\delta_{t}$. Since $f^{-1}(y)$ is a single point we can deform $f$ to a map $g$ such that $g^{-1}(y)=f^{-1}(y), g\left(\delta_{t}\right) \subset \gamma_{t}$ for each $t$, and $g(T \backslash M) \subset$ $T \backslash N$. Also, since the local degree at $f^{-1}(y)$ is \pm 1 we can assume that $g$ is one-to-one on $\delta_{1}$. Moreover, there is a homotopy between $g$ and $f$ which satisfies the conclusion in the lemma that the preimage of $y$ is a single point at each level of the homotopy.

Since $\operatorname{deg}(g)= \pm 1$ it is well known (see for example Theorem 1.1 in [9]) that the relative map $g:(T \backslash \operatorname{int}(M), \partial M) \rightarrow(T \backslash \operatorname{int}(N), \partial N)$ is homotopic, rel boundary, to a map $f^{\prime}$ which is a homeomorphism. Extend to all of $T$ using the constant homotopy from $M$ to $N$ to obtain the desired $f^{\prime}$.

Proposition 3.2. Let $f_{1}, f_{2}: T \rightarrow T$ be homotopic maps where $\left|\operatorname{deg}\left(f_{i}\right)\right|$ $=n$ for $i=1,2$ and $n \neq 0$. If for some $y \in T$, \# $f_{i}^{-1}(y)=n$ for $i=1,2$, then there is a homotopy $H$ between $f_{1}$ and $f_{2}$ such that $\# H(\cdot, t)^{-1}(y)=n$ for all $t \in[0,1]$.

Proof. Consider the $n$-fold covering $p: T^{\prime} \rightarrow T$ which corresponds to the subgroup $f_{i \#}\left(\pi_{1}(T)\right)$. Let $\widetilde{f}_{1}, \widetilde{f}_{2}: T \rightarrow T^{\prime}$ be homotopic lifts of $f_{1}, f_{2}$ respectively. Then $\operatorname{deg}\left(\widetilde{f}_{i}\right)=d$, with $|d|=1$. By construction, for each $i$, the $n$ points in $f_{i}^{-1}(y)$ are mapped by $\widetilde{f}_{i}$ one-to-one onto $p^{-1}(y)$. Moreover, as the Nielsen root number of $f_{i}$ is $n$, the local degree at each such point is $d$. Let $F_{i}$ be homotopies which satisfy the conclusion of Lemma 3.1 with the ends of $F_{i}$ being $\widetilde{f}_{i}$ and $h_{i}$, where $h_{i}$ is a homeomorphism. Now, since $h_{1}, h_{2}$ are homotopic, they are isotopic. Putting together $F_{1}, F_{2}$ and the isotopy yields a homotopy $\widetilde{H}$ between $\widetilde{f}_{1}$ and $\widetilde{f}_{2}$ such that the composition $p \circ \widetilde{H}$ provides the required homotopy $H$. 
Remark. Proposition 3.2 in the case when the degree is \pm 1 also follows from an algebraic result of Nielsen, which is Corollary N4 in Section 3.5 of [7].

As in the proofs of Theorems 1.3 and 1.4 the multiplicative structure of $T$ allows for root problems to be converted into fixed point and coincidence problems. The results for fixed points and coincidences that correspond to Proposition 3.2 are given below in Theorems 3.3 and 3.4. Let $L(f)$ denote the Lefschetz number of the map $f$, and $\Lambda(f, g)$ the Lefschetz coincidence number of the pair $(f, g)$.

TheOREM 3.3. Let $g_{1}, g_{2}: T \rightarrow T$ be homotopic maps with $\left|L\left(g_{i}\right)\right|=n$ for $i=1,2$ and $n \neq 0$. If \# $\mathrm{Fix}\left(g_{i}\right)=n$ for $i=1,2$, then there is a homotopy $H$ between $g_{1}$ and $g_{2}$ such that $\# \operatorname{Fix}(H(\cdot, t))=n$ for all $t \in[0,1]$.

Proof. Consider the maps $h_{i}(x)=g_{i}(x) \cdot x^{-1}$. From [10] we know that the degree of $h_{i}$ is $n$. The proof now follows from Proposition 3.2.

TheOREM 3.4. Let $\left(f_{1}, g_{1}\right),\left(f_{2}, g_{2}\right): T \rightarrow T$ be homotopic pairs of maps with $\left|\Lambda\left(f_{i}, g_{i}\right)\right|=n$ for $i=1,2$ and $n \neq 0$. If $\# \operatorname{Coin}\left(f_{i}, g_{i}\right)=n$ for $i=1,2$ then there is a pair of homotopies $H, G$ between $\left(f_{1}, g_{1}\right)$ and $\left(f_{2}, g_{2}\right)$ such that \#Coin $(H(\cdot, t), G(\cdot, t))=n$ for all $t \in[0,1]$.

4. Minimal coincidences in dimension one. In dimension one the only closed manifold is the 1-torus, which of course is the 1-sphere $S^{1}$.

Proposition 4.1. Let $f_{1}, f_{2}: S^{1} \rightarrow S^{1}$ be homotopic maps. Suppose that $\# f_{i}^{-1}(y)=\left|\operatorname{deg}\left(f_{i}\right)\right|=n$ for $i=1,2$. Then there is a homotopy $H$ between $f_{1}$ and $f_{2}$ such that $\# H(\cdot, t)^{-1}(y)=n$ for all $t \in[0,1]$.

Proof. Although an elementary direct proof can be given, here we observe that when $\operatorname{deg}\left(f_{i}\right) \neq 0$ the proofs of 3.1 and 3.2 given in Section 3 apply to $S^{1}$ as well. Thus we need only consider the case when $\operatorname{deg}\left(f_{i}\right)=0$. Suppose that $\# f_{i}^{-1}(y)=0$ for $i=1,2$. Since $S^{1} \backslash\{y\}$ is contractible we can deform each $f_{i}$ to the constant map at some $c \in S^{1} \backslash\{y\}$. Moreover, throughout the homotopy nothing is mapped to $y$, which establishes the result.

As a consequence we have

TheOREM 4.2. Let $\left(f_{1}, g_{1}\right),\left(f_{2}, g_{2}\right): S^{1} \rightarrow S^{1}$ be homotopic pairs of maps, each pair having $\left|\Lambda\left(f_{i}, g_{i}\right)\right|$ coincidence points. Then there is a pair of homotopies $H, G$ between $\left(f_{1}, g_{1}\right)$ and $\left(f_{2}, g_{2}\right)$ such that \#Coin $(H(\cdot, t), G(\cdot, t))=$ $\left|\Lambda\left(f_{i}, g_{i}\right)\right|$ for all $t \in[0,1]$.

Acknowledgments. The first author would like to thank R. Brown and H. Schirmer for their encouragement and suggestions made in the early stage 
of this work. The second author would like to thank the IME at Universidade de São Paulo for its hospitality during the preparation of this manuscript.

\section{References}

[1] S. Bogatyi, D. L. Gonçalves and H. Zieschang, The minimal number of roots of surface mappings and quadratic equations in free groups, Math. Z. 236 (2001), 419-452.

[2] D. Dimovski and R. Geoghegan, One-paremeter fixed point theory, Forum Math. 2 (1990), 125-154.

[3] R. Geoghegan and A. Nicas, Parametrized Lefschetz-Nielsen fixed point theory and Hochschild homology traces, Amer. J. Math. 116 (1994), 397-446.

[4] D. L. Gonçalves and H. Zieschang, Equations in free groups and coincidence of mappings on surfaces, Math. Z. 237 (2001), 1-29.

[5] J. Jezierski, One codimensional Wecken type theorems, Forum Math. 5 (1993), 421-439.

[6] M. R. Kelly, Some examples concerning homotopies of fixed point free maps, Topology Appl. 37 (1990), 293-297.

[7] W. Magnus, A. Karrass and D. Solitar, Combinatorial Group Theory, Interscience, New York, 1966.

[8] H. Schirmer, Fixed point sets of homotopies, Pacific J. Math. 108 (1983), 191-202.

[9] R. Skora, The degree of a map between surfaces, Math. Ann. 276 (1987), 415-423.

[10] P. Wong, Coincidence of maps into homogeneous spaces, Manuscripta Math. 98 (1999), 243-254.

Departamento de Matemática - IME-USP

Caixa Postal 66281 - Ag. Cidade de São Paulo

CEP: 05315-970

São Paulo, SP Brazil

E-mail: dlgoncal@ime.usp.br
Department of Mathematics and Computer Science Loyola University 6363 St Charles Avenue

New Orleans, LA 70118, U.S.A. E-mail: kelly@loyno.edu

Received 11 December 2000;

in revised form 27 September 2001 